\title{
Assistência farmacêutica no cuidado à saúde na atenção primária: uma revisão integrativa da literatura
}

\author{
Pharmaceutical assistance in health care in primary care: an integrative literature review \\ Asistencia farmacêutica en atención sanitária primaria: revisión integrativa de la literatura
}

Recebido: 05/11/2021 | Revisado: 11/11/2021 | Aceito: 13/11/2021 | Publicado: 17/11/2021

\author{
Maria Fabiana Ferreira de Araújo \\ ORCID: https://orcid.org/0000-0002-8021-5727 \\ Universidade Nilton Lins, Brasil \\ E-mail: marifabaraujo@gmail.com \\ Rodrigues Ferreira de Souza \\ ORCID: https://orcid.org/0000-0001-7617-737X \\ Universidade Paulista, Brasil \\ E-mail: rodriguessouzaferreira@gmail.com \\ Erick Frota Gomes Figueiredo \\ ORCID: https://orcid.org/0000-0002-6127-0544 \\ Universidade Nilton Lins, Brasil \\ E-mail: erick.figueiredo@uniniltonlins.edu.br
}

\begin{abstract}
Resumo
O objetivo do presente estudo foi analisar a importância da assistência farmacêutica no cuidado à saúde dentro da atenção primária, fazendo-se entender o papel primordial do farmacêutico no cuidado centrado no indivíduo e no coletivo. Trata-se de um estudo do tipo revisão integrativa da literatura, descritiva, onde foi pesquisado publicações de cunho científico indexadas nas bases de dados online Biblioteca Virtual em Saúde - BVS (Medline, IBECS e LILACS) e SCIELO dos anos de 2016 a 2021. Foram recuperados 167 artigos, sendo 80 resultados da base de dados MEDLINE, 46 na LILACS, 14 na IBECS, 27 na SCIELO. Após leitura dos títulos e resumos foram selecionados 20 artigos. Observou que a concretização da assistência farmacêutica (AF) é vista como uma das ferramentas para o fortalecimento dentro do Sistema Único de Saúde (SUS). Isso está diretamente ligado ao desenvolvimento da sua gestão participativa no sistema, designo este que garante o acesso aos medicamentos e à integralidade da assistência terapêutica. A inclusão do farmacêutico nesse contexto impacta positivamente na saúde da população quando o mesmo trabalha ao lado da população, cuidado e promovendo saúde, realizando estratégias voltadas para bem-estar do indivíduo e da família.
\end{abstract}

Palavras-chave: Assistência farmacêutica; Atenção primária; Sistema Único de Saúde.

\begin{abstract}
The aim of this study was to analyze the importance of pharmaceutical assistance in health care within primary care, making it possible to understand the primary role of the pharmacist in care centered on the individual and the collective. This is a descriptive, integrative literature review type study, where scientific publications indexed in the online databases Virtual Health Library - VHL (Medline, IBECS and LILACS) and SCIELO from 2016 to 2021 were searched. 167 articles were retrieved, being 80 results from the MEDLINE database, 46 from LILACS, 14 from IBECS, 27 from SCIELO. After reading the titles and abstracts, 20 articles were selected. He noted that the implementation of pharmaceutical care (PA) is seen as one of the tools for strengthening within the Unified Health System (SUS). This is directly linked to the development of its participatory management in the system, which I designate ensures access to medicines and integral therapeutic care. The inclusion of the pharmacist in this context positively impacts the health of the population when he works alongside the population, caring for and promoting health, carrying out strategies aimed at the well-being of the individual and the family.
\end{abstract}

Keywords: Pharmaceutical care; Primary attention; Health Unic System.

\section{Resumen}

El objetivo de este estudio fue analizar la importancia de la asistencia farmacéutica en la atención de la salud dentro de la atención primaria, permitiendo comprender el papel principal del farmacéutico en la atención centrada en el individuo y el colectivo. Se trata de un estudio tipo revisión de literatura descriptiva, integradora, donde se buscaron publicaciones científicas indexadas en las bases de datos en línea Biblioteca Virtual en Salud - VHL (Medline, IBECS y LILACS) y SCIELO de 2016 a 2021. Se recuperaron 167 artículos, siendo 80 resultados de la base de datos MEDLINE, 46 de LILACS, 14 de IBECS, 27 de SCIELO. Después de leer los títulos y resúmenes, se seleccionaron 20 artículos. Señaló que la implementación de la atención farmacéutica (AP) es vista como una de las herramientas para el fortalecimiento dentro del Sistema Único de Salud (SUS). Esto está directamente ligado al desarrollo de su gestión participativa en el sistema, que designo asegura el acceso a los medicamentos y la atención terapéutica integral. La inclusión del farmacéutico en este contexto impacta positivamente en la 
salud de la población cuando trabaja junto a la población, cuidando y promoviendo la salud, llevando a cabo estrategias orientadas al bienestar del individuo y la familia.

Palabras clave: Atención farmacéutica; Atención primaria; Sistema único de Salud.

\section{Introdução}

De acordo com o Ministério da Saúde (MS) e Conselho Nacional de Secretários de Saúde (CONASS) define-se como atenção primária à saúde o agrupamento de ações de saúde no contexto individual e coletivo que compreende a promoção, prevenção, diagnóstico em tempo oportuno, tratamento e reabilitação. Sua construção deve ter como alicerce o exercício de práticas gerenciais e sanitárias, democráticas e participativas realizadas em equipe, direcionadas a populações de territórios bem delineados (BRASIL, 2004).

O termo "Assistência Farmacêutica" teve origem em um cenário focal para o provimento de medicamentos. Mesmo diante do designo da Central de Medicamentos (CEME) mantivesse o termo AF, não existia até a proclamação da Política Nacional de Medicamentos (PNM), um exato conceito de sua finalidade, objetivos e o conjunto de atribuições que a integrasse. Após estruturação da PNM, a AF correspondeu ao planejamento integrado do Sistema Único de Saúde (SUS) permitindo desta forma alicerçar o processo instalado de promoção da cidadania condizentes com os princípios legítimos do direito à saúde (BRASIL, 1971; Bermudez et al., 2018).

Dentro desta construção da PNM houve também a fundação do Programa Nacional de Qualificação da Assistência no Sistema Único de Saúde (Qualifar-SUS). Tratou-se de um plano que teve como intuito ofertar aperfeiçoamento e integração das atividades da $\mathrm{AF}$ nas redes de atenção à saúde. O programa está estruturado em quatro pilares que se constituem entre si, a saber: estrutura, informação, educação e cuidado. (BRASIL, 2015; Rodrigues et al., 2017).

A atenção farmacêutica possui caráter sistemático, multidisciplinar, abrangendo diversas estratégias direcionadas à promoção, proteção e recuperação da saúde do indivíduo e da coletividade, permitindo o acesso e o uso racional dos medicamentos. Neste contexto, entra a importância da atuação do farmacêutico na AF dentro da atenção primária a saúde. Com o passar dos anos, sua inserção na equipe multiprofissional vem ganhando espaço, permitindo que o mesmo assuma papeis de liderança, gerenciamento e o cuidado integral ao cliente (Costa et al., 2017).

Ainda de acordo com o Ministério da Saúde - 2014 no contexto de complexidade do processo saúde-doença-cuidado requer a sistematização do processo de trabalho na Atenção Primária a Saúde - APS, por meio de equipes multiprofissionais, devendo ter uma abordagem interdisciplinar e intersetorial. Fazendo parte desta equipe atua também o farmacêutico quer seja atuando nos Centros de Saúde ou em equipes de referência do Núcleo de Apoio à Família - NASF (BRASIL, 2014a).

É notório ressaltar que nos últimos 19 anos algumas conquistas fortaleceram a atuação do farmacêutico na APS, a saber: a Política Nacional de Medicamentos; a Política Nacional de Assistência Farmacêutica (PNAF/2004); a Política Nacional de Atenção Básica (PNAB/2006), atualizada em 2011; e a publicação da portaria de criação do Núcleo de Apoio à Saúde da Família (NASF) em 2008, atualizada em 2014. Também contribuiu, no âmbito do Ministério da Saúde, a estruturação do Departamento de Assistência Farmacêutica (DAF) em 2003 (BRASIL, 2014b).

A Organização Mundial da Saúde (OMS) fomentou publicações sobre a renovação da atenção primária nas Américas, que inclui um documento sobre os Serviços Farmacêuticos na APS. Esse contexto estimula a implantação de uma nova identidade profissional do farmacêutico para atuar para além da tradicional gestão do medicamento, com abordagem voltada para o cuidado. A integração do farmacêutico na equipe, a formação e o uso de novas tecnologias para atuar na APS e a construção do vínculo com a comunidade e com os usuários são desafios que estão postos (Barberato et al. 2019). 
Neste sentindo este estudo tem por objetivo principal analisar a importância da assistência farmacêutica no cuidado à saúde dentro da atenção primária, fazendo-se entender o papel primordial do farmacêutico no cuidado centrado no indivíduo e no coletivo.

\section{Metodologia}

Trata-se de um estudo de revisão integrativa da literatura, descritiva, onde foi pesquisado publicações de cunho científico indexadas nas bases de dados online Biblioteca Virtual em Saúde - BVS (Medline, IBECS e LILACS) e Scientific Electronic Library Online - SciELO utilizando palavras-chave e espaço temporal compreendidos de 2016 a 2021.

A revisão integrativa da literatura é um método de pesquisa que objetiva traçar uma análise sobre o conhecimento já construído em pesquisas anteriores sobre um determinado tema. Possibilita a síntese de vários estudos já publicados, permitindo a geração de novos conhecimentos, pautados nos resultados apresentados pelas pesquisas anteriores (Botelho et al., 2011). Para a produção, utiliza-se seis etapas, a saber: 1) identificação do tema e seleção da questão de pesquisa, 2) estabelecimento dos critérios de inclusão e exclusão, 3) identificação dos estudos pré-selecionados, 4) categorização dos estudos selecionados, 5) análise e interpretação dos resultados e 6) apresentação da revisão/síntese do conhecimento.

Foram utilizadas obras na língua portuguesa e inglesa com os seguintes descritores em saúde (DeCS): Assistência farmacêutica, Atenção primária à saúde, Sistema Único de Saúde, para responder a seguinte questão de pesquisa: Qual a importância da assistência farmacêutica no cuidado à saúde dentro da atenção primária? Diante disso, foi elaborado o organograma abaixo para organização dos trabalhos encontrados.

Os seguintes critérios de inclusão foram adotados para seleção da produção científica, a saber: a) artigos b) estar disponível em texto completo c) recorte temporal de 2016 a 2021 d) estudos disponíveis nos idiomas português e inglês. Como critérios de exclusão, foram desconsiderados os textos que não tinham relação com a temática ou apresentaram duplicatas. Os artigos após estabelecidos os critérios de inclusão e exclusão foram tabulados cronologicamente em uma planilha do Microsoft Excel 2013.

\section{Resultados}

A partir da busca pelos descritores e filtros, foram recuperados 167 artigos, sendo 80 resultados da base de dados MEDLINE, 46 na LILACS, 14 na IBECS, 27 na SCIELO. Após leitura dos títulos e resumos foram selecionados 19 artigos (Quadro 1) para esta elaboração desta revisão. A Tabela 1 a seguir ilustra a busca realizada.

Tabela 1: Total de artigos selecionados segundo as bases de dados.

\begin{tabular}{|c|c|c|}
\hline Bases de dados & Artigos recuperados & Artigos selecionados \\
\hline MEDLINE & 80 & 4 \\
\hline LILACS & 46 & 5 \\
\hline IBECS & 14 & 8 \\
\hline SciELO & 27 & $\mathbf{1 9}$ \\
\hline Total & $\mathbf{1 6 7}$ & 2 \\
\hline
\end{tabular}

Fonte: Autoria Própria.

Em relação ao ano de publicação dos artigos, compreendidos entre 2016 a 2021, observou-se maior prevalência no ano de 2017 correspondendo a 9 artigos (45\%) das publicações, seguido por 5 artigos publicados em 2020 (30\%), 2 publicados 
em 2016 (10\%), 1 artigo em 2018 (5\%), 1 em 2019 (5\%) e 1 em 2021 (5\%). O gráfico a seguir mostra o percentual segundo o ano de publicação.

Figura 1. Distribuição dos estudos categorizados conforme ano de publicação.

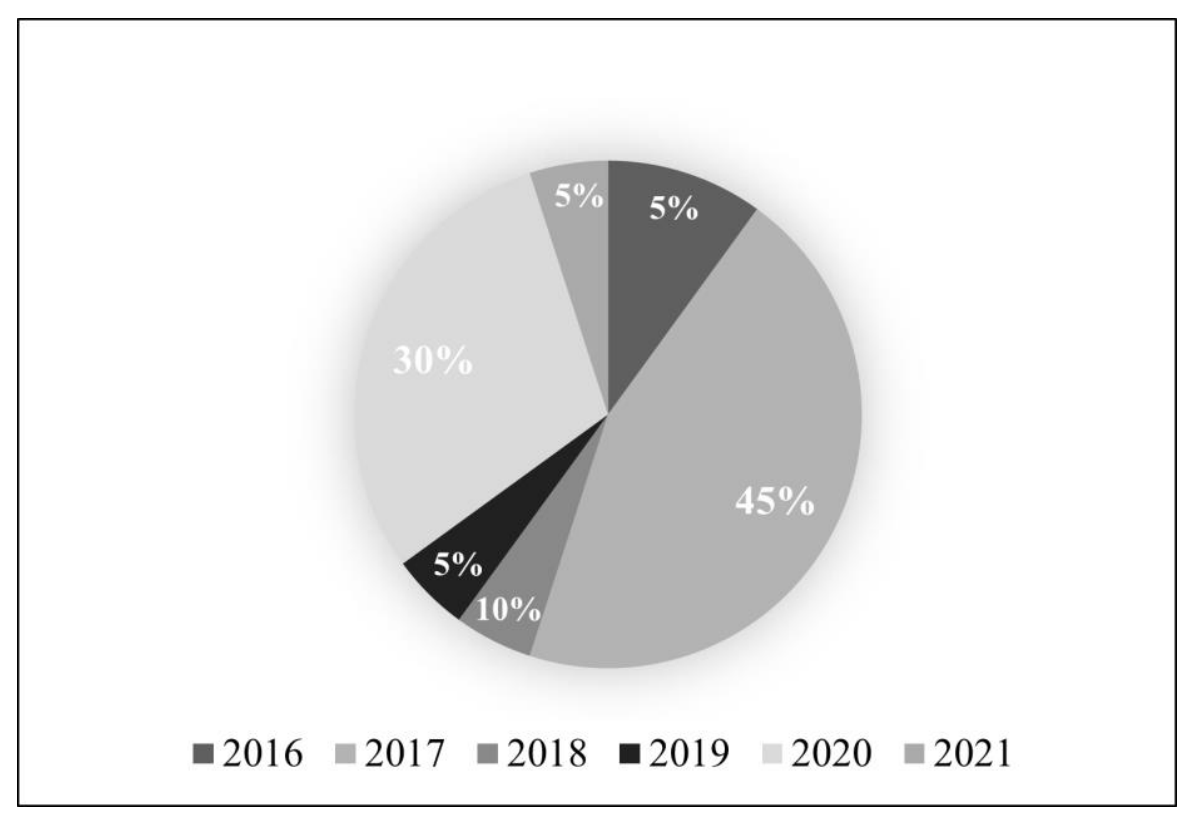

Fonte: Autoria própria

De modo a organizar os artigos de forma cronológica o seguinte quadro abaixo (Quadro 1) foi elaborado pontuando a base de dados, título, autor, ano de publicação, tipo de estudo e abordagem utilizada na pesquisa. 
Quadro 1. Aspectos gerais dos estudos selecionados e características metodológicas.

\begin{tabular}{|c|c|c|c|c|c|}
\hline $\mathbf{n}^{\circ}$ & Bases de Dados & Título & Autor/Ano & Tipo de Estudo & Abordagem \\
\hline 1 & SCIELO & $\begin{array}{l}\text { Assistência farmacêutica no } \\
\text { município do Rio de Janeiro, } \\
\text { Brasil: Evolução em aspectos } \\
\text { selecionados de } 2008 \text { a } 2014\end{array}$ & Silva et al., 2016 & $\begin{array}{l}\text { Longitudinal, } \\
\text { retrospectivo }\end{array}$ & Quantitativo \\
\hline 2 & LILACS & $\begin{array}{l}\text { PNAUM: abordagem integradora } \\
\text { da Assistência Farmacêutica, } \\
\text { Ciência, Tecnologia e Inovação }\end{array}$ & Gadelha et al., 2016 & Documental & Quantitativo \\
\hline 3 & MEDLINE & $\begin{array}{l}\text { Acesso a medicamentos: relações } \\
\text { com a institucionalização da } \\
\text { assistência farmacêutica }\end{array}$ & Barros et al., 2017 & $\begin{array}{l}\text { Transversal, } \\
\text { Exploratório }\end{array}$ & Quantitativo \\
\hline 4 & MEDLINE & $\begin{array}{l}\text { Avanços e desafios da assistência } \\
\text { farmacêutica na atenção primária } \\
\text { no Sistema Único de Saúde }\end{array}$ & Costa et al., 2017 & Narrativa Crítica & Qualitativo \\
\hline 5 & SCIELO & $\begin{array}{c}\text { Atividades farmacêuticas de } \\
\text { natureza clínica na atenção básica } \\
\text { no Bra3sil }\end{array}$ & Araújo, et al., 2017 & $\begin{array}{l}\text { Transversal, } \\
\text { Exploratório }\end{array}$ & Quantitativo \\
\hline 6 & SCIELO & $\begin{array}{l}\text { A contribuição do farmacêutico } \\
\text { para a promoção do acesso } \\
\text { e uso racional de medicamentos } \\
\text { essenciais no SUS }\end{array}$ & Melo et al., 2017 & $\begin{array}{l}\text { Descritivo, } \\
\text { Transversal }\end{array}$ & Quantitativo \\
\hline 7 & SCIELO & $\begin{array}{c}\text { Caracterização da } \\
\text { institucionalização da assistência } \\
\text { farmacêutica na atenção básica no } \\
\text { Brasil }\end{array}$ & Souza et al., 2017 & $\begin{array}{l}\text { Transversal, } \\
\text { Exploratório }\end{array}$ & Quantitativo \\
\hline 8 & SCIELO & $\begin{array}{c}\text { Concepções de assistência } \\
\text { farmacêutica na atenção primária à } \\
\text { saúde, Brasil }\end{array}$ & Costa et al., 2017 & $\begin{array}{l}\text { Descritivo, } \\
\text { Exploratório }\end{array}$ & Quantitativo \\
\hline 9 & MEDLINE & $\begin{array}{l}\text { Financiamento da assistência } \\
\text { farmacêutica na gestão municipal } \\
\text { do Sistema Único de Saúde }\end{array}$ & Faleiros et al., 2017 & $\begin{array}{l}\text { Transversal, } \\
\text { Exploratório }\end{array}$ & Quantitativo \\
\hline 10 & LILACS & $\begin{array}{l}\text { Força de trabalho na assistência } \\
\text { farmacêutica da atenção básica do } \\
\text { SUS, Brasil }\end{array}$ & Carvalho et al., 2017 & Transversal & Quantitativo \\
\hline 11 & LILACS & $\begin{array}{l}\text { Gestão da assistência farmacêutica } \\
\text { na atenção primária no Brasil }\end{array}$ & Gerlack et al., 2017 & $\begin{array}{l}\text { Transversal, } \\
\text { Exploratório }\end{array}$ & Quantitativo \\
\hline 12 & LILACS & $\begin{array}{l}\text { A prática clínica do farmacêutico } \\
\text { no núcleo de apoio à saúde a } \\
\text { família }\end{array}$ & Silva et al., 2018 & $\begin{array}{c}\text { Estudo } \\
\text { autoetnográfico }\end{array}$ & Qualitativo \\
\hline 13 & SCIELO & $\begin{array}{l}\text { Assistência farmacêutica na } \\
\text { atenção básica e Programa } \\
\text { Farmácia Popular: a visão de } \\
\text { gestores de esferas subnacionais do } \\
\text { Sistema Único de Saúde }\end{array}$ & Mattosa et al., 2019 & $\begin{array}{c}\text { Entrevistas } \\
\text { semiestruturadas }\end{array}$ & Qualitativo \\
\hline
\end{tabular}




\begin{tabular}{|c|c|c|c|c|c|}
\hline 14 & SCIELO & $\begin{array}{c}\text { Assistência farmacêutica no } \\
\text { cuidado à saúde na Atenção } \\
\text { Primária: tão perto, tão longe; } 2020\end{array}$ & Maximo et al., 2020 & $\begin{array}{c}\text { Estudo } \\
\text { etnográfico }\end{array}$ & Qualitativo \\
\hline 15 & LILACS & $\begin{array}{l}\text { Ética e assistência farmacêutica na } \\
\text { atenção básica: desafios cotidianos; } \\
2020\end{array}$ & Molina et al., 2020 & $\begin{array}{c}\text { Exploratório- } \\
\text { descritivo }\end{array}$ & Qualitativo \\
\hline 16 & SCIELO & $\begin{array}{l}\text { Percepções de atores sociais sobre } \\
\text { Assistência Farmacêutica na } \\
\text { atenção primária: a lacuna do } \\
\text { cuidado farmacêutico }\end{array}$ & Soares et al., 2020 & $\begin{array}{c}\text { Estudo } \\
\text { exploratório }\end{array}$ & Qualitativo \\
\hline 17 & IBECS & $\begin{array}{c}\text { Primary Health Care and } \\
\text { Community Pharmacy in Ireland: a } \\
\text { lot of visions but little progress }\end{array}$ & Henman et al., 2020 & Documental & Quantitativo \\
\hline 18 & IBECS & $\begin{array}{l}\text { Primary health care policy and } \\
\text { vision for community pharmacy } \\
\text { and pharmacists in Colombia }\end{array}$ & Amariles, et al., 2020 & Documental & Quantitativo \\
\hline 19 & MEDLINE & $\begin{array}{l}\text { Implementation of pharmaceutical } \\
\text { services in Brazilian primary } \\
\text { health care: a crosssectional study }\end{array}$ & Pereira et al., 2021 & $\begin{array}{c}\text { Estudo } \\
\text { transversal }\end{array}$ & Quantitativo \\
\hline
\end{tabular}

Fonte: Autoria própria.

\section{Discussão}

\subsection{Desafios da assistência farmacêutica na atenção primária a saúde}

Nas últimas 30 décadas, diversas eventualidades corroboraram para o desenvolvimento da AF na atenção primária a saúde, dos quais podemos citar a Publicação da Política Nacional de Medicamentos (PNM), Publicação das Diretrizes Curriculares do Curso de Farmácia e definição do farmacêutico generalista com formação voltada ao SUS, Elaboração do Consenso Brasileiro de Atenção Farmacêutica e Criação do Programa Nacional de Qualificação da Assistência Farmacêutica no Sistema Único de Saúde (QualiFar-SUS) isso conforme o levantamento feito na pesquisa de Soares; Brito; Galato, 2020.

Observou que a concretização da assistência farmacêutica (AF) é vista como uma das ferramentas para o fortalecimento dentro do Sistema Único de Saúde (SUS). Isso está diretamente ligado ao desenvolvimento da sua gestão participativa no sistema, designo este que garante o acesso aos medicamentos e à integralidade da assistência terapêutica. Contudo, o que se tem observado nos últimos anos nos municípios brasileiros é uma frágil descentralização das ações e à capacidade de gestão da AF nesses municípios, assim apontou o estudo feito por Gerlack et al., 2017.

De acordo ainda com a pesquisa de Gerlack et al., 2017, é preocupando o fato de que um quarto dos municípios do território brasileiro não apresenta a AF estruturada conforme as normativas das secretarias Municipais de saúde (SMS), fato este que pode prejudicar sua inserção no processo de planejamento em saúde e ressoar negativamente na execução de suas atribuições.

Dados da pesquisa de Gadelha et al., 2016 sobre a implantação da AF nos territórios brasileiros discorre sobre o conceito da AF segundo a Resolução que promulgou a PNAF - Política Nacional de Assistência Farmacêutica que define a AF como um agrupamento de atitudes de promoção, proteção e recuperação da saúde, individual e coletivo, empregando o uso de medicamentos como matéria-prima primordial, objetivando ao seu acesso e uso prudente. 
Na pesquisa realizada por Costa et al., 2017 apontam para os avanços que a AF vem mostrando em parecia com atores envolvidos nesse processo de instituição dessa política nos municípios brasileiros, mesmo diante dos obstáculos que precisam ser observados, debatidos e enfrentados pela sociedade.

Costa et al., 2017 também faz menção sobre à estrutura dos serviços farmacêuticos onde observou-se significativa presença de sistemas informatizados para a gestão da AF nos municípios, ressaltando como ponto positivo para o desenvolvimento do serviço. No entanto, a dificuldade está na sua integração em rede com diferentes serviços de saúde. Observado isso, se faz necessário, garantir condições mais oportunas aos ambientes em que se realizam esses serviços, quer seja pertinente a infraestrutura, tempo de espera para o atendimento nas farmácias.

Em um estudo realizado por Barros et al., 2017 em municípios brasileiros evidenciou que o sistema informatizado na gestão da AF compreendia um total de 55,6\% dos pacientes que pegavam medicamentos em unidades que não dispunha de um sistema para organizar a distribuição dos medicamentos. Onde havia sistema que compreendeu 18,5\% estava ligado à rede de outras unidades, tornando um ponto negativo no processo de vinculação das informações.

Nos resultados encontrados por Faleiros et al., 2017 sobre descentralização da AF mostrou que segundo os secretários municipais, cerca de $54 \%$ dos municípios brasileiros apoiaram o regime de descentralização parcial na forma de união da execução dos insumos do Componente Básico da Assistência Farmacêutica - CBAF. A coordenação da AF dispunha com total emancipação da gestão dos recursos financeiros definidos para a AF em 22,7\% dos municípios e parcial em 45,6\%. Dos municípios estudados o total de 67,3\% fazia gastos com a organização da AF.

O estudo de Faleiros et al., 2017 trouxe ainda sobre o repasse financeiro destinados a AF, onde mais da metade dos municípios brasileiros recebeu no ano anterior à pesquisa, recursos oriundos dos estados ou da União designado à estruturação e organização da AF. Mais de 50\% dos municípios fizeram algum tipo de gasto com a Estruturação da AF em seus territórios.

Na pesquisa realizada por Souza et al., 2017 sobre institucionalização da AF mostrou que um panorama heterogêneo de formação da AF nas regiões brasileiras. A dimensão das estruturas formais foi a que melhor de sobressaiu. A legitimação da AF no organograma reconhece o seu desempenho diante as demais áreas públicas, fazendo-se perceptível à população como um todo e aos demais segmentos da saúde.

Souza et al., 2017 pontua sobre a informatização da AF assim como Barros et al., 2017 também mostrou. Ainda que no Brasil, as taxas dos que afirmam possuir sistema informatizado estarem acima de $70 \%$, observou-se significativas diferenças entre as regiões, mostrando as desigualdades regionais de infraestrutura e logística dos serviços de saúde. Um exemplo a ser elencado sobre isso é a baixa adesão do uso do sistema Hórus, no qual há fortíssimas evidências de que esse sistema fortalece o controle e o monitoramento na utilização dos medicamentos.

Costa et al., 2017 buscou-se entender o conceito de AF na visão dos farmacêuticos. Os resultados revelaram a elevada incidência de uma visão geral de que a AF está centrada no medicamento, assim como, na ideia com o cuidado com os usuários de medicamentos.

Em um estudo recente realizado por Pereira et al., 2021 sobre a implementação da assistência farmacêutica na atenção básica no Brasil e serviços farmacêuticos (PharmSes), observou-se que este serviço se encontra em um nível grave. Ainda é preocupante quando se examinou as dimensões e componentes do PharmSes. A implantação do PharmSes iniciou em 1988 e ainda está em andamento. Surgiu com a substituição para a instalação de uma nova responsabilidade municipal, estabelecida pela Política Nacional de Medicamento (PNM).

Carvalho et al., 2017 estudou sobre Força de trabalho na assistência farmacêutica da atenção básica do SUS, Brasil e evidenciou que a força de trabalho na assistência farmacêutica era predominantemente composta por mulheres, na faixa etária de 18 a 39 anos, com formação superior (90,7\% na coordenação e 45,5\% na unidade de dispensação), com vínculo 
empregatício efetivo (concursado), há mais de um ano no cargo ou atividade e jornada de trabalho semanal superior a 30 horas, tanto na gestão municipal quanto nas unidades de dispensação de medicamentos.

Na pesquisa realizada por Matos et al., 2019 sobre a AF na ABS na visão de gestores observaram os problemas relacionados a AF havendo concordância sobre a deficiência no financiamento. Destacaram a falta de correção do valor de repasse federal no elemento básico de financiamento da $\mathrm{AF}$, a discrepância entre o financiamento e o aumento do serviço local elevada pela expansão das políticas de $\mathrm{AB}$ e $\mathrm{AF}$, o aumento de preços dos medicamentos e a expansão das relações.

Máximo; Andreazza; Cecilio, 2020 realizou um estudo de natureza etnográfica com profissionais farmacêuticos de 7 UBS de 3 municípios sobre a AF na atenção básica. O estudo revelou o potencial que AF traz de perspectiva para dentro da UBS. É importante uma atuação mais dialogada entre os profissionais, em particular os médicos que foram estudados no presente trabalho e a equipe de saúde que observam algo que não ocorre de forma tão mecânica quanto pensávamos.

\subsection{Processo de trabalho do farmacêutico no cuidado à saúde na APS e dentro da equipe multiprofissional}

É um desafio trabalhar em uma equipe multiprofissional, principalmente para o farmacêutico, pois se trata de uma estratégia relativamente inovadora que se deu na APS a partir de 2009. O objetivo é fortalecer uma cultura de partilha de saberes e relacionamento horizontal e igualitário entre os profissionais. O estímulo do trabalho multiprofissional para o farmacêutico assim descreveu Silva et al., 2018 é ter a compreensão da sua responsabilidade especifica ao atuar em uma equipe de saúde, para assim realizar o compartilhar de saberes e das responsabilidades comuns.

Silva et al., 2018 em sua pesquisa descreve sobre o processo de trabalho do farmacêutico dentro APS, fazendo uma divisão de trabalho farmacêutico em técnico-gerencial e técnico-assistencial para possibilitar uma grande carga de emoções negativas nos farmacêuticos. Esse tópico foi um dos pontos mais questionado em diário de campo e entrevistas desta pesquisa. Sentimentos como ansiedade, angústia, esgotamento, interrupções constantes, preocupações, cansaço, sufocamento e frustação foram elencados pelos entrevistados.

Molina; Hoffmann; Finkler 2020 narraram as dificuldades enfrentadas pelos farmacêuticos na APS, principalmente no Núcleo de Apoio de Saúde da Família - NASF, apontando a pulverização das equipes. Um ponto destacado foi a própria estrutura de alguns centros de saúde, que não ajuda na interação entre os profissionais, interferindo na integração positiva para um trabalho colaborativo e participativo. Foi elencado outras problemáticas que corrobora para a fragmentação do cuidado, a saber, a falta de integração entre a ABS e as farmácias, é provável que um centro de saúde dispense medicamentos sem compreender que se remete a uma pessoa com HIV por exemplo. Observa-se que as farmácias da ABS não estão em concordância e planejamento para atender junto com as esquipes da saúde da família - ESF.

Um estudo realizado em São Paulo por Melo \& Castro, 2017 sobre a contribuição do farmacêutico na promoção da utilização coerente dos medicamentos no SUS apontou sobre as intervenções do farmacêutico junto aos prescritores para mudanças no padrão de prescrição, foram ocultos pelo prescritores, pois não se admitia a aprovação do gestor nem a autenticidade do papel do farmacêutico em questões que transcendiam o aspecto logístico em relação ao medicamento.

Araújo et al., 2017 observou que a maioria dos farmacêuticos de sua pesquisa declararam realizar atividades de natureza clínica mesmo não dispondo de local específico para atuar, condição esta primordial à proteção da privacidade e confidencialidade nas atividades com os usuários. Problemáticas em relação aos recursos financeiros foi levantado pelos entrevistados, onde indicaram realizar certa improvisação que demanda esforço dos profissionais, além de não contarem com critérios que racionalizam a oferta dessas atividades nos serviços de saúde.

Amariles; Ceballos; González-Giraldo, 2020 realizaram uma pesquisa sobre o papel do farmacêutico na ABS na Colômbia. Nesse cenário, sob a ótica da farmácia, as diretrizes do MS e Proteção Social sobre a APS fazem parte a assistência 
farmacêutica. Contudo, não fica claro as competências dos farmacêuticos ou da farmácia comunitária. Os farmacêuticos estão envolvidos apenas nas redes de serviços de saúde especializados ou de apoio no modelo de APS.

Outro estudo realizado na Irlanda por Henman, 2020 discorreu sobre a importância da farmácia popular e o papel do farmacêutico no cuidado a saúde dentro da APS e chegaram à conclusão de que tanto a farmácia comunitária como a APS, o sistema público-privado do serviço procede em aumento de custos, fragmentação da prestação de serviços e concorrência para pacientes privados. A APS é vista como uma coleção de serviços com base em agendamento e muito poucos dados são reunidos rotineiramente para fiscalizar o uso desses serviços por pacientes particulares e públicos.

Silva et al., 2016 já enfatizava em sua pesquisa que se faz necessário ampliar a incorporação de profissionais farmacêuticos nas equipes das unidades pelo território brasileiros, ofertando mais ainda o serviço desse profissional. Isso é de suma importância visto que, as evidências apontam uma eficiente gestão técnica dos processos logísticos dos medicamentos, bem como a relação desses profissionais no cuidado, corroborando para a melhoria do uso certo de medicamentos ofertados na APS.

\section{Considerações Finais}

O presente trabalho buscou reunir as evidências científicas dos últimos 5 anos de publicação sobre a assistência farmacêutica na atenção primária a saúde - APS, assim como o papel do profissional farmacêutico e suas atribuições dentro da equipe multiprofissional. Observou-se com isso, a evolução da assistência farmacêutica em todo território brasileiro mesmo diante dos desafios. A inclusão do farmacêutico nesse contexto impacta positivamente na saúde da população quando trabalha ao lado da população, cuidando e promovendo saúde, realizando estratégias voltadas para bem-estar do indivíduo e da família.

A assistência farmacêutica se mostrou no decorrer dos anos institucionalizada, descentralizadas nos municípios do Brasil. A informatização dos sistemas vem crescendo melhorando assim o atendimento como um todo ao usuário do SUS. Com o avanço da gestão da AF, o acesso e uso racional dos medicamentos ocorrerá de forma satisfatória e o farmacêutico poderá consolidar suas intervenções voltadas ao cuidado tanto na prática clínica na APS como na gestão participativa dentro da equipe multiprofissional.

Espera-se que a partir das evidências aqui levantadas se possa contribuir para o conhecimento profissional do farmacêutico, além de enfatizar a necessidade de crescente busca por atualizações e mais estudos que possam agregar a abordagem teórica com a prática clínica, fazendo-se entender também a importância da assistência farmacêutica na atenção básica a saúde e seu papel primordial na equipe multiprofissional no cuidado a saúde do indivíduo e da coletividade.

\section{Referências}

Amariles, P., Ceballos, M., \& Gonzalez-Giraldo, C. (2020). Primary health care policy and vision for community pharmacy and pharmacists in Colombia. Pharmacy Pract (Granada), Redondela, 18(4) 2159. doi.org/10.18549/pharmpract.2020.4.2159.

Araújo, P.S. et al. (2017). Atividades farmacêuticas de natureza clínica na atenção básica no Brasil. rev. Saude Publica. 51 Supl 2:6s. doi.org/10.11606/S15188787.201705100710.

Barberato L. C., Scherer M. D. A., \& Lacourt R. M. C. (2019). O farmacêutico na atenção primária no Brasil: uma inserção em construção. Ciência \& Saúde Coletiva, 24(10), 3717-26.

BARROS, R.D. et al. (2017). Acesso a medicamentos: relações com a institucionalização da assistência farmacêutica. rev. Saude Publica. 51 Supl 2:8s. doi.org/10.11606/S1518-8787.2017051007138.

Bermudez J. Á. Z. et al. (2018). Assistência Farmacêutica nos 30 anos do SUS na perspectiva da integralidade. Ciência \& Saúde Coletiva, $23(6), 1937-51$.

Botelho L. L. R., Cunha C. C. A., \& Macedo M. (2011). The integrative review method in organizational studies. Gestão e Sociedade, 5(11), $121-136$.

Brasil. (2014b). Cuidado Farmacêutico na atenção básica. Caderno 1: Serviços Farmacêuticos na Atenção Básica à Saúde [Internet]. Vol. 1. Brasília: MS. <https://bvsms.saude.gov.br/bvs/publicacoes/servicos_farmaceuticos_atencao_basica_saude.pdf>. 
Brasil. (2004). Conselho Nacional de Secretários de Saúde - CONASS. Atenção Primária. Seminário para a estruturação de consensos. Caderno de informação técnica e memória de Progestores. Brasília: CONASS. < http://189.28.128.100/dab/docs/geral/conass_documenta2.pdf>.

Brasil. (1971). Presidência da República. Decreto no 68.806, de 25 de Junho de 1971. Institui a Central de Medicamentos (CEME). Diário Oficial da União; 26 jun, 4(1), 4839.

Brasil. (2014a). Ministério da Saúde. Secretaria de Atenção à Saúde. Departamento de Atenção Básica. Núcleo de Apoio à Saúde da Família / Ministério da Saúde, Secretaria de Atenção à Saúde, Departamento de Atenção Básica. - Brasília: Ministério da Saúde.

Brasil. (2015). Ministério da Saúde. Secretaria de Ciência, Tecnologia e Insumos Estratégicos. Departamento de Assistência Farmacêutica Insumos Estratégicos. QUALIFAR-SUS: eixo estrutura - atenção básica - instruções técnicas. Brasília, DF: Ministério da Saúde.

Carvalho, M. N. et al. (2017). Força de trabalho na assistência farmacêutica da atenção básica do SUS, Brasil. rev. Saude Publica. 51 Supl 2:16s. doi.org/10.11606/S1518-8787.2017051007110.

Costa, E. A. et al. (2017). Concepções de assistência farmacêutica na atenção primária à saúde, Brasil. Rev Saude Publica. 51 Supl 2:5s. doi.org/10.11606/S1518-8787.201705100710.

Costa, K. S. et al. (2017). Avanços e desafios da assistência farmacêutica na atenção primária no Sistema Único de Saúde. rev. Saude Publica. 51 Supl 2:3s. doi.org/10.11606/S1518-8787.2017051007146.

Faleiros, D. R. et al. (2017). Financiamento da assistência farmacêutica na gestão municipal do Sistema Único de Saúde. rev. Saude Publica. 51 Supl 2:14s. doi.org/10.11606/S1518-8787.2017051007060.

Gadelha, C. A. G. et al. (2016). PNAUM: abordagem integradora da Assistência Farmacêutica, Ciência, Tecnologia e Inovação. rev. Saúde Pública. 50 Supl 2:3s. doi: 10.1590/S1518-8787.2016050006153.

Gerlack, L. F. et al. (2017). Gestão da assistência farmacêutica na atenção primária no Brasil. rev. Saude Publica. 51 Supl 2:15s. doi.org/10.11606/S15188787.2017051007063

Henman, M. C. (2020). Primary Health Care and Community Pharmacy in Ireland: a lot of visions but little progress. Pharmacy Pract (Granada), Redondela, $18(4), 2224$.

Matos, L. et al. (2019). Assistência farmacêutica na atenção básica e Programa Farmácia Popular: a visão de gestores de esferas subnacionais do Sistema Único de Saúde. rev. Saúde Soc. São Paulo, 28(1), 287-298.

Maximo, S. A., Andreazza, R., \& Cecilio, L. C. O. (2020). Assistência farmacêutica no cuidado à saúde na Atenção Primária: tão perto, tão longe. Physis: Revista de Saúde Coletiva, Rio de Janeiro, 30(1), 300107.

Melo, D. O., \& Castro, L. L. C. (2017). A contribuição do farmacêutico para a promoção do acesso e uso racional de medicamentos essenciais no SUS. Ciência \& Saúde Coletiva, 22(1), 235-244.

Molina, L. R., Hoffmann, J. B., \& Finkler, M. (2020). Ética e assistência farmacêutica na atenção básica: desafios cotidianos. rev. Bioét, 28(2), 365-375.

Pereira, N. C. et al. (2021). Implementação da assistência farmacêutica na atenção primária à saúde no Brasil: um estudo transversal. BMC Family Practicehttps, 22(170), 2-11.

Rodrigues P. S., Cruz M. S., \& Tavares N. U. L. (2017). Avaliação da implantação do Eixo Estrutura do Programa Nacional de Qualificação da Assistência Farmacêutica no SUS. rev. Saúde e Debate. 41(n.especial), 192-208.

Silva, D. A. M. et al. (2018). A prática clínica do farmacêutico no núcleo de apoio à saúde a família. Trab. Educ. Saúde, 16(2), 659-682.

Silva, R. M. et al. (2016). Assistência farmacêutica no município do Rio de Janeiro, Brasil: evolução em aspectos selecionados de 2008 a 2014 . Ciência \& Saúde Coletiva, 21(5), 1421-32.

Soares, L. S. S., Brito, E. S., \& Galato, D. (2020). Percepções de atores sociais sobre Assistência Farmacêutica na atenção primária: a lacuna do cuidado farmacêutico. rev. Saúde debate, 44(125), 411-426.

Souza, G. S. et al. (2017). Caracterização da institucionalização da assistência farmacêutica na atenção básica no Brasil. rev. Saude Publica. 51 Supl 2:7s. doi.org/10.11606/S1518-8787.201705100713. 